\title{
Framing - Zur Rolle von Deutungsmustern in Medien
}

\author{
Ist Greta Thunberg eine Heldin oder ein Opfer? Eine Frage des Framings.
}

\author{
von Prof. Dr. Urs Dahinden
}

Ist die 16-jährige schwedische Schülerin Greta Thunberg eine Heldin, weil sie (ohne ein politisches Amt oder eine Führungsfunktion in einem Umweltverband) die «Schulstreiks für das Klima» und die daraus entstandene internationale Bewegung „Fridays for Future“ (FFF) initiiert hat? Oder ist sie ein bedauernswertes Opfer, ein Kind ohne Kindheit, das von ihren Eltern wie eine Marionette gesteuert und für politische Zwecke instrumentalisiert wird? Diese Fragen sollen hier nicht diskutiert und abschließend beantwortet werden. Jede Antwort auf diese Fragen verlangt aber nach einer Deutung und Interpretation der unbestrittenen Fakten über Greta Thunberg. Die dabei verwendeten Deutungsmuster werden in der Kommunikationswissenschaft als Frames (Rahmen) bezeichnet, und der Prozess, in dem diese Deutungsmuster erstellt und verbreitet werden als Framing (Rahmung). Das Beispiel von Greta Thunberg macht deutlich, dass in öffentlichen Debatten über kontroverse Themen meist kein Konsens über das angemessene Framing herrscht, sondern dass sich die Kontoverse genau um diese Frage dreht: Welches Framing ist bei diesem Thema angebracht? Es herrscht mit anderen Worten ein Framing-Wettkampf.

\section{Welche Elemente gehören zu einem Frame?}

In einer oft zitierten Definition des U.S.-Amerikaners Robert M. Entman (1993) werden die folgenden vier Elemente von Frames genannt:

Frames bieten eine Problemdefinition, die verbunden ist mit einer Ursachenzuschreibung. Des Weiteren wird eine Bewertung des Problems abgegeben, die auf moralischen oder anderen Werten beruhen kann und auch mit einer Handlungsempfehlung zur Lösung des Problems verbunden ist. In der Medienberichterstattung werden diese vier Frame-Elemente meist nicht explizit und vollständig ausgeführt, sondern es reicht, wenn ein oder zwei Punkte davon erwähnt werden. Da das Medienpublikum die Frames als Grundmuster bereits kennt, ergänzt es automatisch die fehlenden Elemente. Auch die erwähnten Namen dieser Frames (David-Goliath, Ohnmacht) werden in der Medienberichterstattung kaum genannt, sondern hauptsächlich in der Kommunikationswissenschaft als Fachbegriffe verwendet (Dahinden 2006, S. 210).

\section{Greta Thunberg als Heldin: David-Goliath-Frame}

Diese allgemeine Definition soll am Beispiel von Greta Thunberg und ihrer Darstellung als Heldin erläutert werden: Die Problemdefinition in diesem David-Goliath-Frame (Dahinden 2006, S. 286) ist ein asymmetrischer Konflikt zwischen einer schwachen Partei (David = Greta) und einem übermächtigen Gegner (Goliath = aktuelle Klimapolitik). Die Ursache des Problems lässt sich als Machtmissbrauch der stärkeren Seite beschreiben. Die aktuelle Politik nimmt das Klimaproblem zu wenig ernst und ergreift keine wirksamen Gegenmaßnahmen.

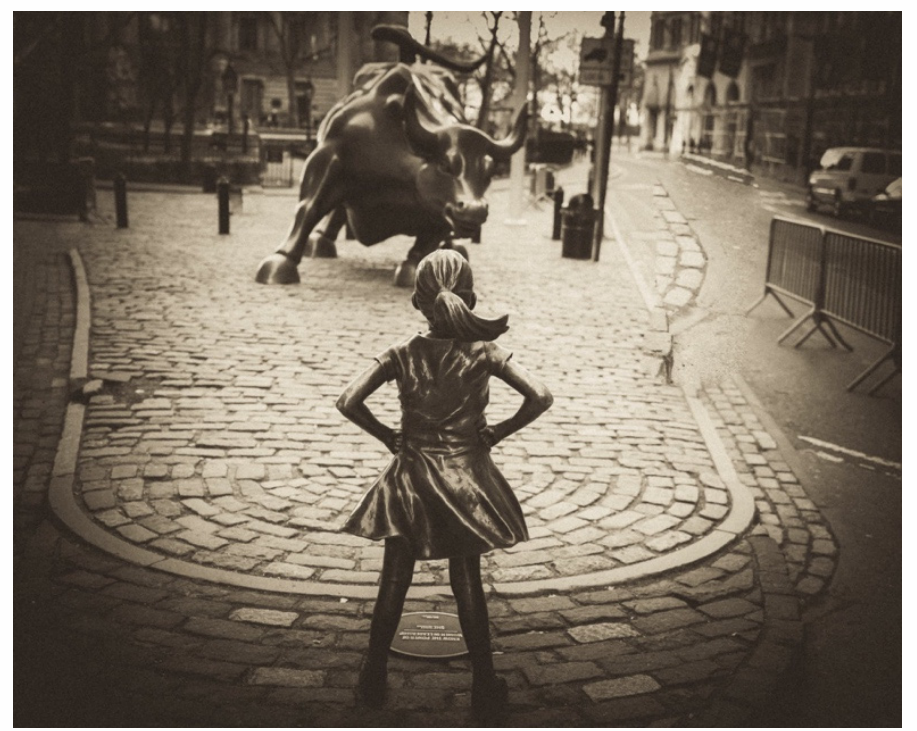

Abbildung 1. Skulptur "Fearless Girl" der Bildhauerin Kristen Visbal (Foto: William Canady von Pexels)

Die Bewertungstendenz innerhalb des Frames läuft auf ein positives Urteil gegenüber der schwächeren und ein negatives gegenüber der stärkeren Partei hinaus. David, bzw. Greta genießen unsere Sympathie und werden schlussendlich auch siegen. Die Handlungsempfehlung kann hier als eine Ermutigung von schwachen Parteien verstanden werden, im Sinne, dass sich ein Widerstand lohnt, auch wenn kein Gleichgewicht der Kräfte vorhanden ist.

\section{Greta Thunberg als Opfer: Ohnmacht-Frame}

Wird Greta Thunberg dagegen als Opfer in einem Ohnmacht-Frame (Dahinden 2006, S. 286) dargestellt, so sind die Frame-Elemente vollständig anders festgelegt: Die Problemdefinition ist nicht mehr die Klimapolitik, sondern die private Familienproblematik einer Minderjährigen, welche zusätzlich noch durch eine Krankheit (Asperger-Syndrom) belastet ist. Die Ursache des Problems sind ihre Eltern und ihre soziale Umgebung, welche sie für Ihre umweltpolitischen Zwecke instrumentalisieren. Die Bewertung des Problems, nämlich ihr Engagement für die Klimapolitik, ist keine bewundernswürdige Leistung, sondern nur eine Folge von elterlicher Manipulation oder Zwang. Auch die Unterstützung ihres politischen Engagements durch die Eltern wird negativ bewertet. Die Handlungsempfehlung läuft daraus hinaus, dass die Eltern ihr Erziehungsverhalten ändern sollten, um Greta eine normale Kindheit zu ermöglichen.
ISSN 2629-5784 Transfe rlus - Aktue lle Beiträge zur Medienbildung In Transfer ${ }^{\text {Plus }}$ besp rechen Autoren aus Wissensc haft und Forschung leicht verständlich und prägnant aktuelle Befunde aus Journalismus, Medien und Gesellschaf t. Literatur- und Medientippsgeben Anregungen zu Vertiefung und Thematisierung in der Bild ung sarbeit. Transfer rius wird auf Qucosa, dem sä chsisc hen OpenAccess-Dokumenten- und Publikationsserver publiziert.

\section{Redaktion}

Dr. Benjamin Big I

Prof. Dr. Denise Sommer 


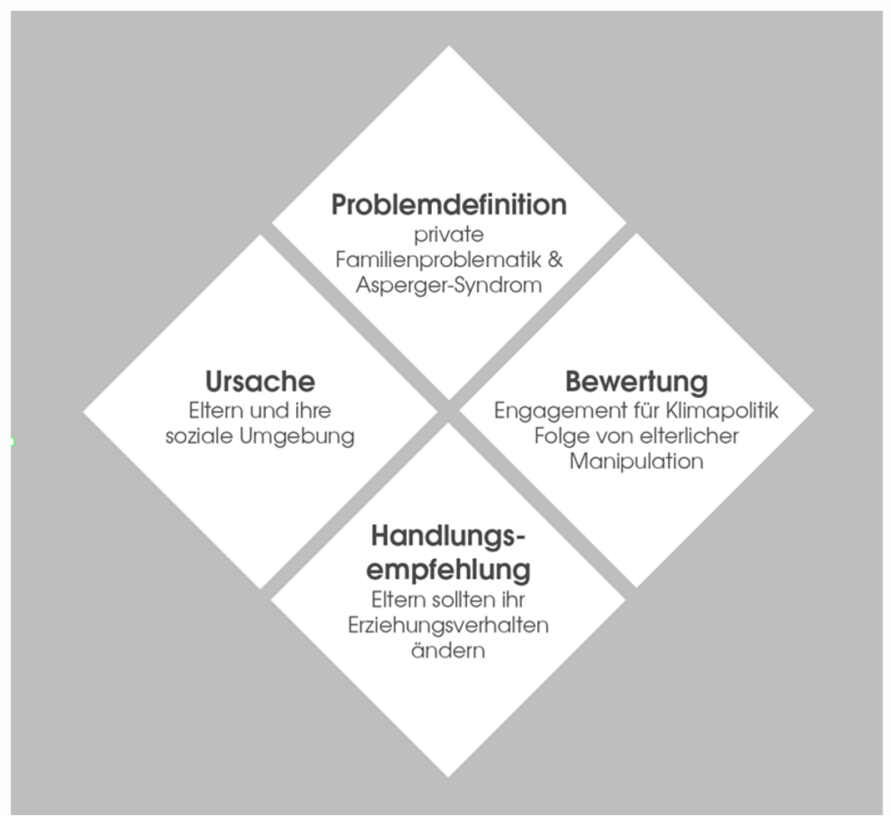

Abbildung 2. Die vier Frame-Elemente des Ohnmacht-Frames in der Berichterstattung über Greta Thunberg

\section{Ist Framing etwas Negatives,}

\section{das Medien möglichst vermieden sollten?}

Nein, Framing ist aus einer medienethischen Perspektive nicht abzulehnen als irreführende Manipulation, sondern entspricht der gewünschten Funktion, dass Medien die Komplexität der modernen Welt für das Medienpublikum reduzieren. Allerdings sollten Medien sich bei ihrem Framing an zwei Leitlinien orientieren: Erstens sollten Medien ein einseitiges Framing vermeiden und Frame-Vielfalt in ihren Publikationen fördern. Zweitens sollten Medien sich um Transparenz und Meta-Kommunikation über FramingProzesse bemühen. Welche Akteure fördern mit welchen Interessen und mit welchen Begriffen (Klimakatastrophe vs. Klimalüge) ein bestimmtes Framing?

Mit Hilfe dieser Transparenz über Framing-Prozesse erhält das Medienpublikum die notwendigen Hinweise, um seine eigene Medienkompetenz weiter zu entwickeln, das Kommunikationsverhalten von allen Beteiligten kritisch analysieren zu können und sich schlussendlich auch eine eigene Meinung darüber bilden zu können.

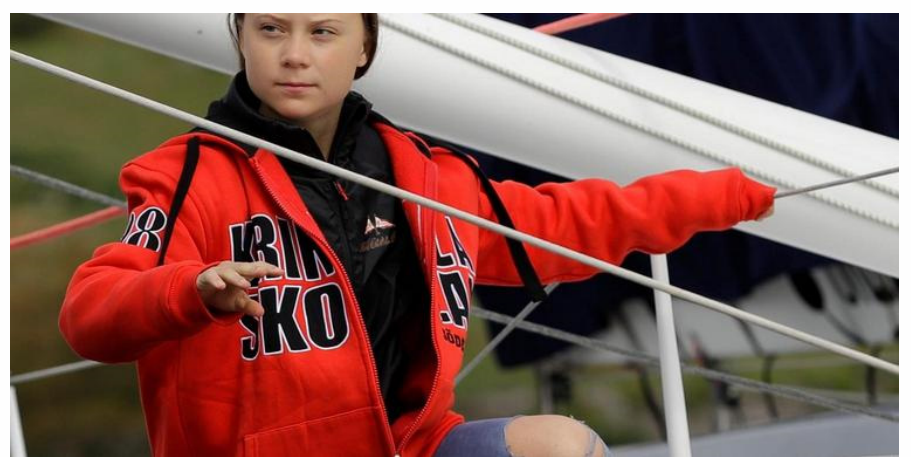

Abbildung 3. Greta Thunberg während eines Pressetermins (Foto: Kirsty Wigglesworth/AP/dpa)

\section{Take-home-message}

- Wir können nicht nicht-framen. Medien wie auch alle anderen an der öffentlichen Kommunikation Beteiligten sollen Framing nicht vermeiden, sondern sich vielmehr um Frame-Vielfalt bemühen.

- Frames und die dahinterliegenden Framing-Prozesse werden meist nicht explizit genannt und dargestellt. Medien sollten sich deshalb darum bemühen, Transparenz und Metakommunikation über Framing herzustellen.

- Medienkompetente Bürgerinnen und Bürger, Pädagogen und Jugendliche sollten Framing-Bemühungen erkennen und in der Lage sein, diese kritisch einzuordnen.

\section{Quellen}

Dahinden, U. (2006). Framing - Eine integrative Theorie der Massenkommunikation. (Forschungsfeld Kommunikation, Band 22). Köln: Herbert von Halem Verlag.

Entman, R. M. (1993). Framing: Toward Clarification of a Fractured Paradigm. Journal of Communication, 43(4), 51-58. https://doi.org/10.1111/j.1460-2466.1993.tb01304.x

\section{Autor der Ausgabe}

Urs Dahinden ist Professor für Kommunikations- und Medienwissenschaft an der Fachhochschule Graubünden in Chur (Schweiz). Kontakt: urs.dahinden@fhgr.ch

\section{Empfohlene Zitation}

Dahinden, U. (2019). Framing - Zur Rolle von Deutungsmustern in Medien. In: B. Bigl (Hrsg.). TransferPLUS - Beiträge zur Medienbildung. Issue 05/2019. https://nbn-resol-

ving.org/urn:nbn:de:bsz:14-qucosa2-358474
Macht der Worte

Framing in 3 Minuten erklärt

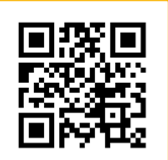

Was ist politisches Framing? logo! Erklärt (ZDF tivi)
ARD-Papier zum Framing

sorgt für Diskussion

ZAPP (NDR)

\section{Literaturempiehlungen}

Dahinden, U. (2006). Framing - Eine integrative Theorie der Massenkommunikation. (Forschungsfeld Kommunikation, Band 22). Köln: Herbert von Halem Verlag. Matthes, J. (2019). Framing (2., aktualisierte Auflage). Baden-Baden: Nomos.

Wehling, E. (2018). Politisches Framing: Wie eine Nation sich ihr Denken einredet - und daraus Politik macht. Berlin: Ullstein Taschenbuch Verlag. 